\title{
Inverted left atrial appendage: a complication of de-airing during cardiac surgery
}

\author{
Arindam Choudhury, MD (D) - Jitin Narula, MD • Pankaj Kumar, MD • \\ Shivani Aggarwal, MD • Usha Kiran, MD
}

Received: 12 May 2015/ Accepted: 1 June 2015/Published online: 3 July 2015

(C) Canadian Anesthesiologists' Society 2015

A male patient with a bicuspid aortic valve associated with severe aortic stenosis and a dilated aortic root underwent a Bentall procedure. Apart from the aortic pathology, no other abnormalities were detected by a preoperative transesophageal echocardiography (TEE) examination. After an otherwise uncomplicated surgical procedure, the patient's heart was deaired using standard maneuvers, including manual cardiac agitation and conventional aspiration of the aortic root vent and left ventricular vent inserted during the operation via the superior pulmonary vein. After the de-airing, a hypoechoic structure suggestive of atrial thrombus was seen in the left atrium (LA) using TEE (Figure A).

The surgeon was informed of the unusual finding, and on direct visual inspection of the heart, it was confirmed that the left atrial appendage (LAA) had become intussuscepted and inverted inside the LA. Weaning from cardiopulmonary bypass (CPB) was attempted in order to re-establish normal LA pressures and thus revert the appendage to its normal position. Nevertheless, this attempt was unsuccessful, and application of a Valsalva maneuver also failed. Accordingly, during a brief return to CPB, the tip of the LAA was grasped with Russian tissue forceps and reverted to its normal anatomic position, which was confirmed with a repeat TEE examination (Figure B).

A. Choudhury, MD $(\bowtie) \cdot$ J. Narula, MD · P. Kumar, MD . S. Aggarwal, MD · U. Kiran, MD

Department of Cardiac Anaesthesiology, All India Institute of Medical Sciences, Ansari Nagar, New Delhi, India e-mail: archymd@yahoo.com
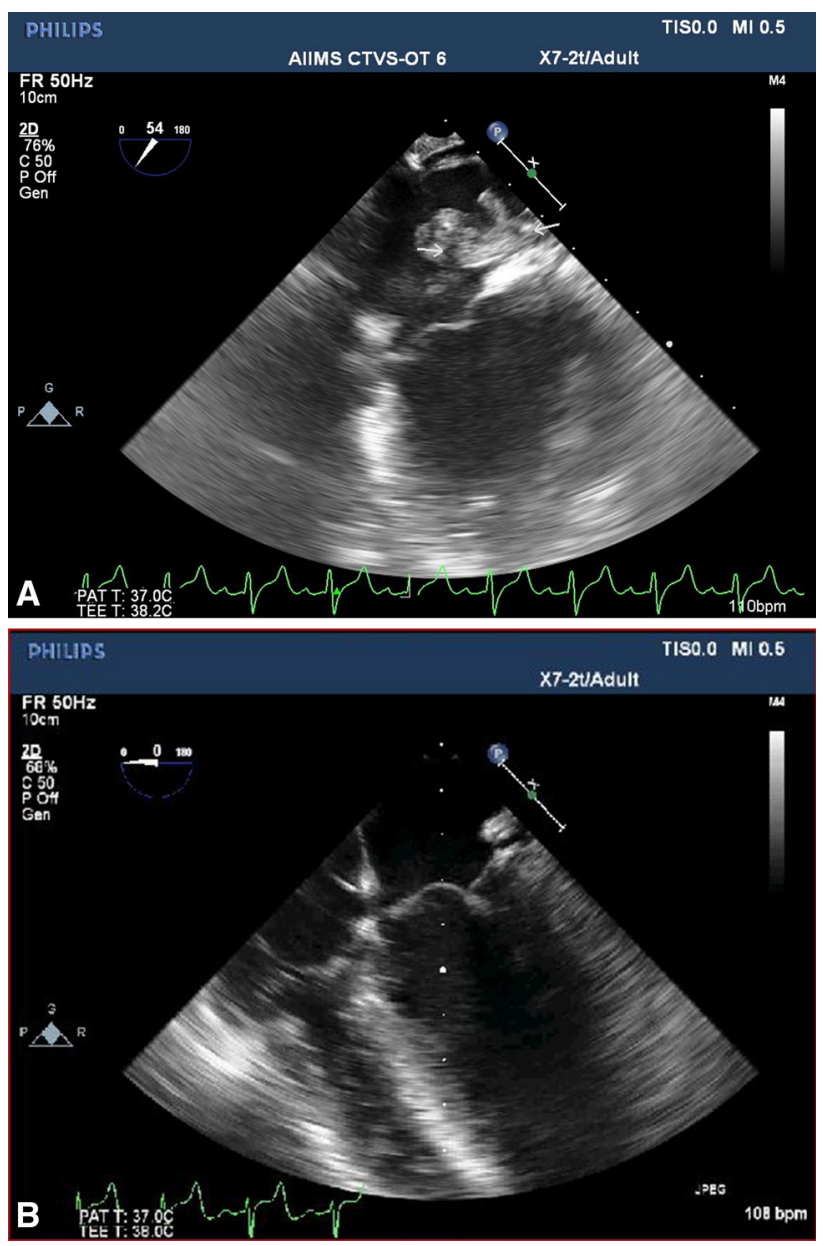

Figure (A) Inverted left atrial appendage ME4C view (arrow). (B) Normal LAA after reduction of intussusception. 
Although inversion of the LAA appears to be a relatively benign surgical phenomenon, there is a potential for complications, such as necrosis and rupture, that can lead to pericardial tamponade ${ }^{1}$ as well as obstruction to left ventricular inflow at the mitral valve. ${ }^{2}$ The possible cause for the intussusception was likely due to excessive negative pressure applied to the left ventricular vent, either during the procedure or with its removal, or possibly by digital insertion during the de-airing maneuvers. The sustained intussusception of the LAA, even after weaning from CPB, could have been due to a relatively narrow neck of the LAA. ${ }^{3}$ A post-procedure TEE examination can facilitate early detection and rapid correction of this complication.
Conflicts of interest None declared.

\section{References}

1. Koepfli $P$, Enseleit F, Jenni R. Mysterious floating structure in the left atrium after coronary artery bypass grafting. Cardiovascular Medicine 2007; 10: 153.

2. Molaei A, Tabib A, Meraji M, Shemirani $R H$. Inverted left atrial appendage: a cause of left ventricular inflow obstruction. Iranian Cardiovascular Research Journal 2010; 4: 139-41.

3. Arya VK, Channabasappa LV, Thingnam SK. A mysterious echogenic structure in the left atrium after coronary artery bypass graft surgery. J Cardiothorac Vasc Anesth 2009; 23: 914-5. 